\title{
Manufacturing of high precision aspherical and freeform optics
}

\author{
André M. Hoogstrate*a ${ }^{*}$ Casper van Drunen ${ }^{a}$, Bart van Venrooy ${ }^{\mathrm{a}}$, Rens Henselmans ${ }^{\text {a }}$ \\ ${ }^{a}$ TNO, Stieltjesweg 1, 2628 CK, Delft, The Netherlands
}

\begin{abstract}
Aspherical and freeform optical elements have a large potential in reducing optical aberrations and to reduce the number of elements in complex high performance optical systems. However, manufacturing a single piece or a small series of aspherical and freeform optics has for long been limited by the lack of flexible metrology tools. With the cooperative development of the NANOMEFOS metrology tool by TNO, TU/e and VSL, we are able to measure the form of aspheres and freeforms up to $500 \mathrm{~mm}$ in diameter with an accuracy better than $10 \mathrm{~nm}$ rms. This development opened the possibility to exploit a number of iterative, corrective manufacturing chains in which manufacturing technologies such as Single Point Diamond Turning, freeform grinding, deterministic polishing and classical polishing are combined in an iterative loop with metrology tools to measure form deviation (like CMM, LVDT contact measurement, interferometry and NANOMEFOS).

This paper discusses the potentials, limitations and differences of iterative manufacturing chains used by TNO in the manufacturing of high performance optics for astronomical purposes such as the manufacturing of the L2 of the Optical Tube Assembly of the four laser-guide star facility of the ESO VLT, Manufacturing of Aluminum freeform mirrors for the SCUBA-2 instrument. Based on these results we will give an outlook into the new challenges and solutions in manufacturing high-precision optics.
\end{abstract}

Keywords: Polishing, SPDT, Asphere, Freeform, NANOMEFOS

\section{INTRODUCTION}

One of the focal points of the TNO department Instrument Manufacturing is the manufacturing of ultra-precision asperical and freeform optical parts for a wide range of astronomical, and space based earth observation instruments. To serve a broad range of materials, Polishing processes, mainly for glass-type materials and and Single Point Diamond Turning (SPDT), mainly for non-ferrous materials, are available. Typical single components or small series ( $<5$ parts) are required by the customer and therefore accurate measurements of these optics is challenging. TNO has, together with the TU/e and VSL developed and build the NANOFOS, a metrology tool able to measure spherical, aspherical and freeform optics. This paper introduces the various fabrication strategies for aspheric and freeform optics available at TNO, together with some illustrative examples of the results so far.

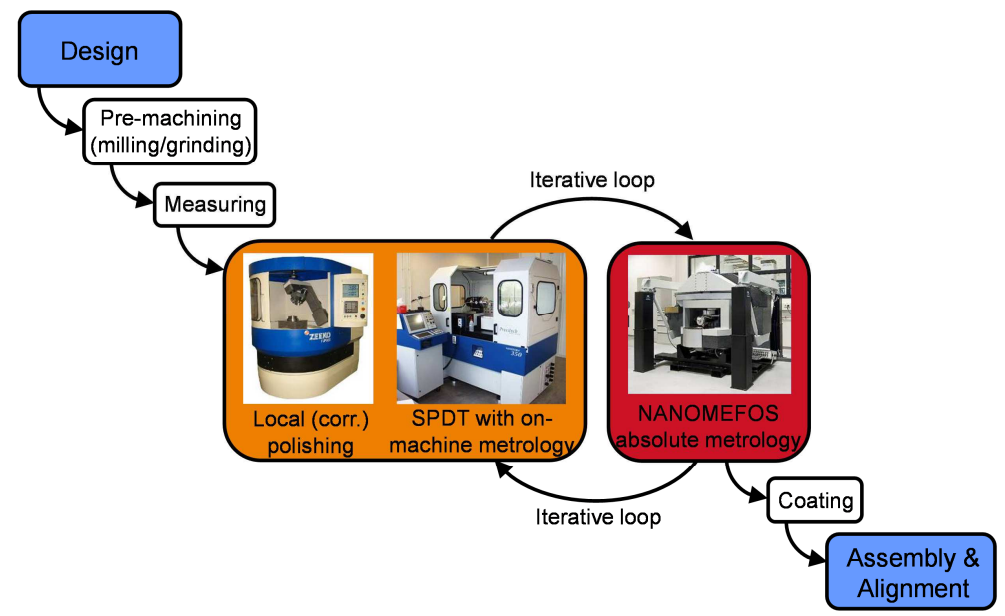

Figure 1: Generic process chain for ultra-precision aspherical or freeform optical manufacturing processes at TNO

Modern Technologies in Space- and Ground-based Telescopes and Instrumentation II, edited by Ramón Navarro, Colin R. Cunningham, Eric Prieto, Proc. of SPIE Vol. 8450, 84502Q

(C) 2012 SPIE · CCC code: $0277-786 X / 12 / \$ 18 \cdot$ doi: $10.1117 / 12.926040$

Proc. of SPIE Vol. $845084502 Q-1$ 


\section{FREEFORM FABRICATON STRATEGIES}

The generic process chain for ultra-precision optical components is shown in Figure 1. After design, pre-machining and pre-measuring of the component TNO has the ability to finish the optical surface by either a sub-aperture CNC controlled polishing process or to generate the final surface with single point diamond turning. For metrology purposes there is the ability to use on-machine metrology for the SPDT process or to use the NANOMEFOS. For spherical and flat surfaces a standard interferometer is also available.

Characteristic for the freeform fabrication strategy is the iterative loop between the manufacturing and measurement processes. The benefits of this approach are best visible in the manufacturing of single pieces or small series of aspheric or freeform components, where the development of special tooling or measurement equipment is economically not viable.

\section{ITERATIVE MANUFACTURING}

Conventional lens polishing technologies using classical lapping technologies always have had an iterative character. Control of the figure is realized by the geometry of the polisher: how the lap is stroked and the table with the optic is rotated. The part has to be measured regularly, usually with interferometry, to adjust the fabrication process to correct the errors in the surface. This iteration between manufacturing and measurement results in a time consuming process. Simultaneously the cost of the optic is highly dependent on the efficiency of the optician in converging to the final specification[1]. With the increasing popularity of aspheric and freeform optics, which are difficult to manufacture both due to the limits in classical manufacturing technologies and available measurement technologies, new manufacturing and measurement technologies had to be developed.

Aspheric and freeform manufacturing is a different from classical production technologies in that manufacturing is performed using sub-aperture tools. These tools are significantly smaller than the optical area to be machined, thus allowing the shaping of aspheres and freeforms. The sub-aperture approach also enables the local variation of machining parameters, like local positioning, local feed or dwell-time. When correctly applied the local variations can be used to correctively machine an optical surface. The correction must be based on an error map, resolving local errors at least one order better than the corrections, which can be resolved by the manufacturing process. Examples of deterministic machining processes include diamond turning, computer-controlled polishing (CCP), ion-beam figuring, plasma-jet etching magneto-rheological finishing and fluid jet polishing.

Although it is sometimes postulated that every error, which can be measured, also can be corrected, there are limitations in the achievable results with correction. The next chapters deals with the possibilities and limitations of the various iterative loops available at TNO.

\section{EQUIPMENT AVAILABLE AT TNO}

\subsection{Equipment for deterministic polishing}

TNO applies a Zeeko Intelligent Robot Polisher (IRP) for computer controlled polishing. A rotating inflatable bonnet is used as a tool, which is covered with a polishing cloth. We use commercially available polishing slurries and polishing clothes. Our robot (FJP600) is capable of polishing large optics up to $600 \mathrm{~mm}$. However, this can only be achieved in spiral polishing mode. For raster polishing, the machine is limited to approximately $300 \mathrm{~mm}$. Figure 2 shows a picture of the equipment. 

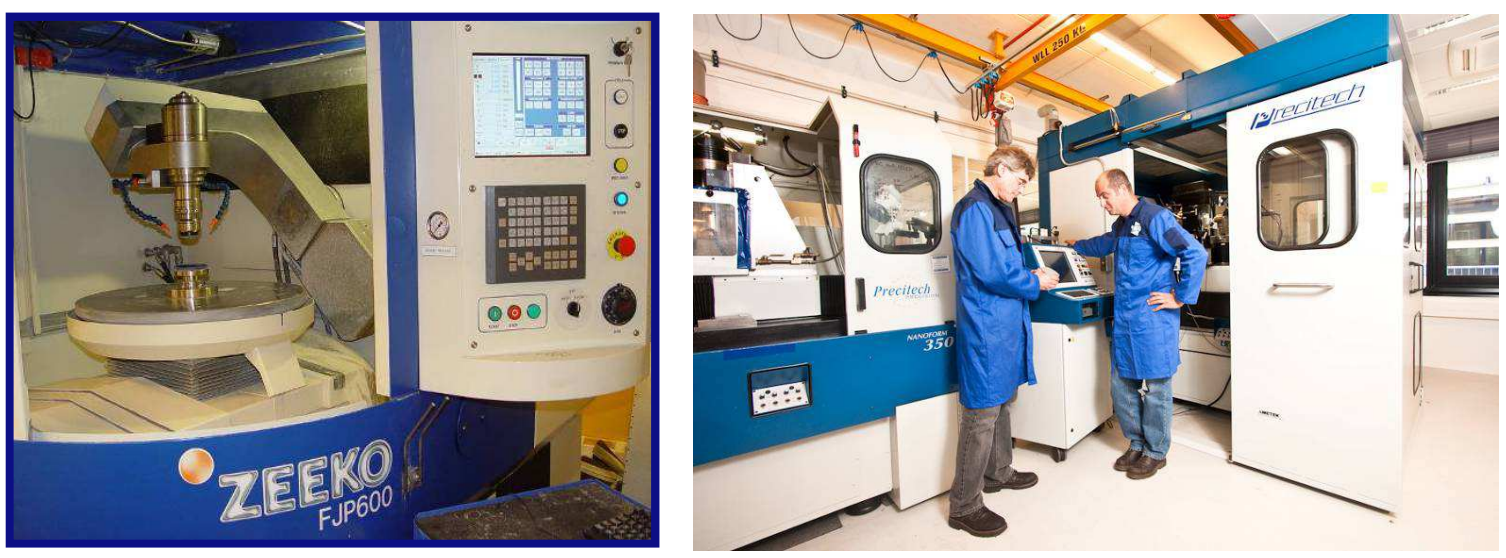

Figure 2: Zeeko FJP600 polishing robot (left). Nanoform 350 and Freeform 700 SPDT-machines (right)

\subsection{Equipment for SPDT}

Two SPDT machines from Precitech are available, a Nanoform 350 and a Freeform 700. Both machines are based on two linear hydrostatic oil-bearing slides $(X, Z)$ in a T-configuration. The Freeform 700 is additionally equipped with a linear hydrostatic oil-bearing slide as $\mathrm{Y}$-axis, mounted on de $\mathrm{X}$-axis and with a rotary hydrostatic oil-bearing table used as a B-axis, mounted on the Z-axis. On both machines an air-bearing main spindle is mounted which can also act as a rotary $\mathrm{C}$-axis.

Both machines are equipped with on-machine metrology in the form of a LVDT-contact measurement, which, in conjunction with the linear axis can be used to measure the optical surface with high accuracy. These measurements can be used to create an 1D or 2D error-map of the machined surface. Figure 2 shows the setup of these machines.

\subsection{Measurement of aspheric and freeform surfaces}

When on-machine metrology is not enough to measure an aspherical or freeform optic (which is often the case), TNO employs a new and very promising instrument called NANOMEFOS [2]. This instrument is a non-contact measuring machine for aspherical and freeform optics up to $500 \mathrm{~mm}$ diameter. It has been developed by TNO, Eindhoven University of Technology and the Dutch metrology institute VSL, funded within the framework of the Dutch Innovationoriented Research Programme (IOP).

When using NANOMEFOS, the surface to be measured is placed on a continuously rotating air-bearing spindle, while a specially developed optical probe is positioned over it by a motion system (see Figure 3). The optical probe facilitates high scanning speeds (up to $1.5 \mathrm{~m} / \mathrm{s}$ ), and its $5 \mathrm{~mm}$ measurement range captures the non-rotational symmetry of the surface. This allows for the stages to be stationary during the measurement of a circular track, reducing the dynamically moving mass to $45 \mathrm{~g}$. The position of the probe is measured interferometrically relative to a silicon carbide metrology frame. Capacitive probes measure the spindle position, also relative to this reference frame. Static as well as dynamic position errors from this short metrology loop are compensated for in data processing. 


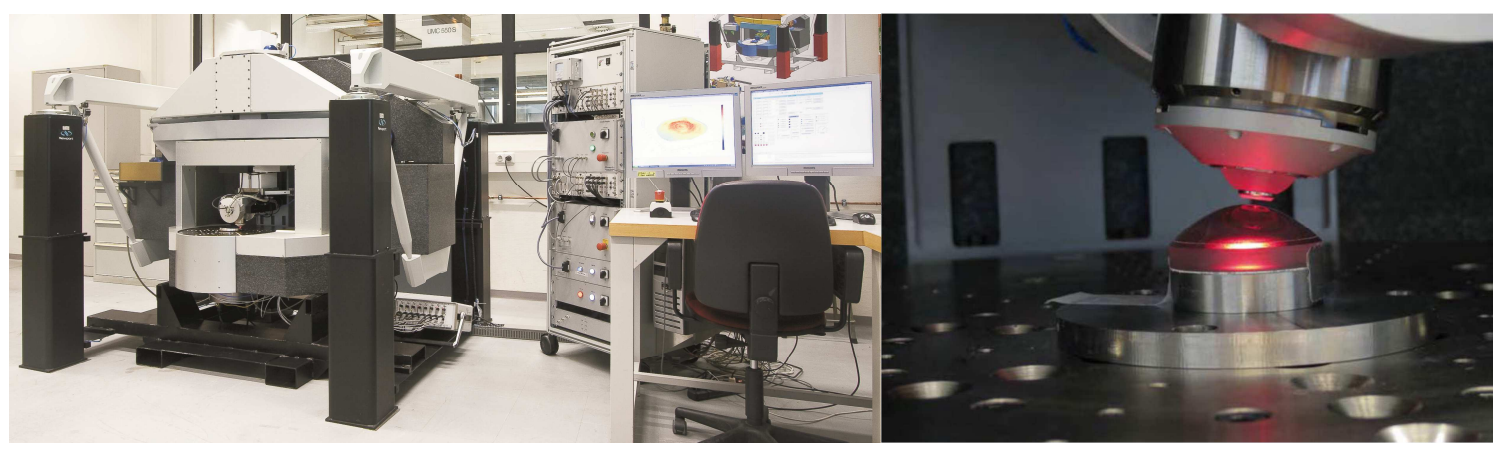

Figure 3: Overview of the NANOMEFOS setup (left). Close-up of the measurement head in action (right)

Reproducibility tests have shown that a reproducibility of approximately $1-3 \mathrm{~nm} \mathrm{rms}$ can be reached. The machine has recently been calibrated, resulting in a form measurement uncertainty of approximately 10-30 nm rms, depending on the surface type. The major advantage of NANOMEFOS is its flexibility. Measuring an asphere or freeform conventionally is difficult and generally requires the use of a dedicated setup with e.g. a computer-generated hologram. However, with NANOMEFOS every asphere can be programmed and measured easily, without the need for custom-made (if at all possible) computer-generated holograms. For aspheres, the departure from the best-fit-sphere is unlimited, and for freeforms the maximum allowed non-rotational symmetry is $5 \mathrm{~mm} \mathrm{PV}$. The slope may range from $-45^{\circ}$ concave to $90^{\circ}$ convex (i.e. cylindrical). This means that large convex optics are also easily measured. Typically, a highly curved convex optic of > $50 \mathrm{~mm}$ diameter cannot be measured on a standard 4" interferometer and requires large-aperture interferometers, which is why convex aspheres are not frequently applied. Another benefit of the machine is that it measured absolute radius of curvature, to sub- $\mu \mathrm{m}$ sag accuracy levels. The NANOMEFOS machine therefore removes boundaries of application of aspheres and freeforms, enabling much more freedom in an optical design.

\section{ITERATIVE MACHINING}

\subsection{Computer controlled sub-aperture polishing}

Computer controlled sub-aperture polishing has a two-folded goal. In most applications the optical surface is premachined close to its final shape. The polishing process has to meet the final shape specifications, and simultaneously meet the required surface roughness. Depending on the initial conditions of the surface, both roughness and form-error.

Bonnet-based sub-aperture polishing is a dwell-time driven material removal process. By varying the local feed-speed over the surface, the local material removal can be controlled to reduce form errors. Small height variations in the order of a few micrometers will have a negligible influence on the spot size and shape of the polishing aperture and on the local material removal. Consequently, the requirement for position accuracy are relaxed and instead of ultra-precision machine tools, common machine tool designs can be used. Additionally, small, elastic workpiece deformations due to mounting induced stresses will have no influence on the local material removal and hence on the error correction.

For first polishing runs the material removal is assumed to be proportional related to the feed speed over the surface. For high precision polishing the variation in local removal on various locations as function of location and slope angle angles must be taken into account. Therefor on a number of locations and under various slope angles the material removal is predetermined. This knowledge is convoluted with the error map to obtain a more precise dwell-time calculation for the bonnet on the surface.

In figure $4 \mathrm{a}$ it is clear that the material removal in the polishing spot is not uniformly distributed. A vertical line pattern is visible, which is caused by the rotation of the bonnet around its axis. If the feed speed is in the direction of this line pattern extreme mid-spacial frequencies are introduced (figure 4b). The sine-waved pattern has an amplitude of $80 \mathrm{~nm}$ in this case and Rq is $24 \mathrm{~nm}$. However, if the feed speed is perpendicular to the line pattern these lines get smeared out, resulting in a much lower roughness of $1.6 \mathrm{~nm}$. This becomes clear in figure 4c where a track spacing of $1 \mathrm{~mm}$ was applied to get a removal of $1 \mu \mathrm{m}$. High spatial frequencies become visible for this case, but they can be optimized since the spatial length is the ratio of the used feed rate $(\mathrm{mm} / \mathrm{min})$ and tool rotation speed (rev/min). 


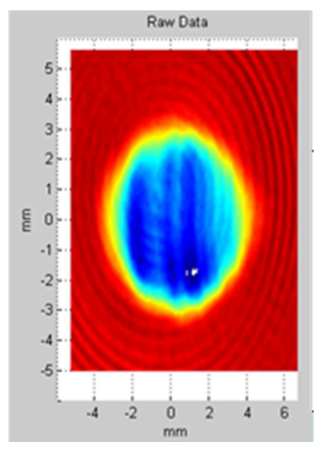

(a) Polishing spot

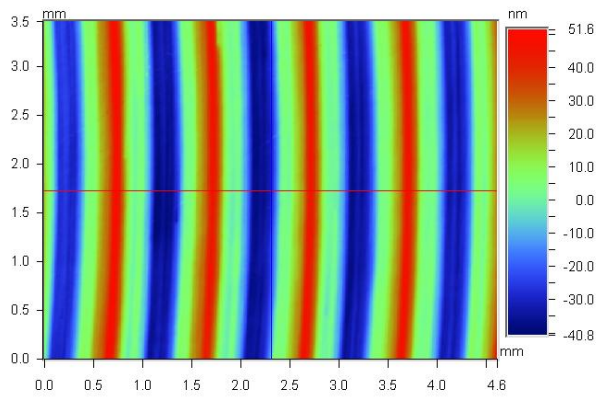

(b) $\mathrm{Rq}=24 \mathrm{~nm}$ on $4.6 \times 3.5 \mathrm{~mm}$ area

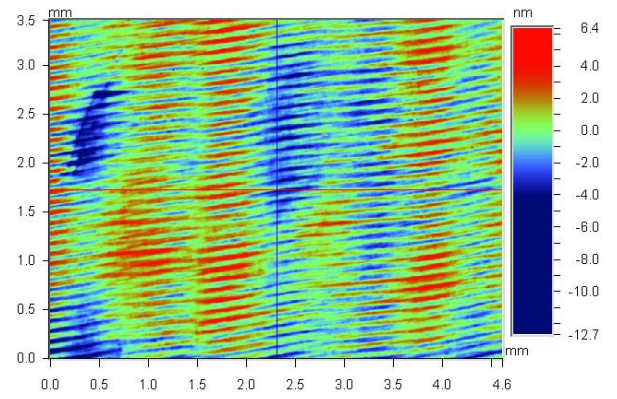

(c) $\mathrm{Rq}=1.6 \mathrm{~nm}$ on $4.6 \times 3.5 \mathrm{~mm}$ area

Figure 4: Polishing spot (a) and typical mid-spatial patterns obtained with (b) bonnet rotation and feed speed in the same direction and (c) with bonnet rotation and feed speed orthogonal to each other.

Iterative machining with identical process conditions and measuring the result has the advantage that the local material removal can be measured accurately, which can be used as an input for more precise dwell time calculations. In this case iterative machining require a stable and repeatable polishing process. Stability is however limited by the wear of the bonnet and the properties of the slurry. Especially for larger optics this requires a careful selection of process parameters to be able to finish the number of required iterative process steps with a single bonnet.

The amount of achievable correction in a single step is at one side determined by the process conditions, and maximum achievable feed speed variation. The first being controlled by e.g. the selected abrasives, polishing cloth and bonnet pressure. The latter by machine-acceleration limitations in combination with the workpiece geometry. These interactions forces us to develop a tailored iterative polishing scheme for each dedicated aspheric or freeform optic. This scheme is based on the pre-machined condition of the surface, machine simulations, dry-runs and polishing experiments. The scheme must ensure that the process in a defined number of iterations will convolute to the required specifications.

Conclusively we can state that the challenges in NC controlled sub-aperture polishing are the selection of polishing parameters and to maintain these parameters in a stable way. Additional challenges are introduced with large optics in the form of increased importance of wear and with heavily curved optics in the form of acceleration limits of the machine.

\subsection{Corrective manufacturing with SPDT}

SPDT, in contrast to polishing is basically not a dwell-time related material removal process, but a geometric-driven material removal process. SPDT generates with every single run a complete new surface. Normally the depth of cut is in the range of a few microns, therefore deviations below 1 micron, introduced in a previous cutting run, will have no influence on the current run. This is to say, there is no inheritance of previous surface errors. SPDT is therefore not a real iterative process, but rather a corrective process. Deviations from the nominal surface in SPDT are contributed by errors in setup, in motion, in clamping and in tooling.

Surface errors due to setup are largely caused by a radial offset between the zero-point of the tool and the true rotation axis of the main spindle. This type of errors is most easily corrected using a spherical test-workpiece, as this type of error has no direct relation with the asphere or freeform to be manufactured. The radial shift can be determined using onmachine metrology, making the shift compensation a rather fast iteration cycle. The thermal balance in the machine should be stable and identical during setup and machining of the optical surface, else machine deformations due to thermal effects can still cause radial offset and radial offset drift.

Deviations of the optical surface caused by motion errors, which are in turn related to straightness and position errors of the axis, will repeat well between successive runs, and can be corrected in an corrective machining cycle. The selection between on-machine and off-machine measurement is a difficult trade-off. Aspheres can be measured relatively fast and with good accuracy with on-machine metrology. Repeatability of measurement results in the order of $30 \mathrm{~nm}$ PV are not uncommon. The measurements of freeforms is at the other side a slow process. Off-line measurement with NANOMEFOS might be faster and more accurate, however, unmounting and remounting might also introduce additional 
positioning errors. Usually, an intermediate body is therefore applied that can be easily interchanged between the machines.

Temperature fluctuations in the machine environment, either induced by thermal sources in the machine or external influences will limit de achievable level of correction. Thermal effects will cause mechanical deformations of the whole setup, in turn leading to motion errors between tool and workpiece. These motion errors will not repeat between successive machining runs. In the worst-case error correction based on the previous run will introduce additional form errors instead of reducing them.

In contrast with polishing, mounting-induced workpiece deformations are copied one-to-one in the surface error. So even when a part is machined perfectly, internal stresses due to clamping will be released when the part is unclamped and the surface will still have errors. Moreover the fixation in SPDT must be better as compared to polishing, due to the high rotational speed of the workpiece. Theoretical these errors can be compensated for with an error-map measured with the part in unclamped situation. However, similar internal stress distributions between successive runs are a prerequisite, which is nearly impossible to realize in practice. Our efforts focus on stress-free clamping of the optics for SPDT. In most cases interfacing is realized in two step. The optic is mounted as stress-free as possible on an product-specific interface, which is in turn clamped on a generic interface on the machine. This allows for easy removal of the mirrorinterface assembly for off-machine metrology measurements of the mirror.

During the toolpath generation the tooltip is assumed to have a perfect spherical shape with known diameter. But in practice the tool radius will locally vary. This tool radius variation, called tool waviness, will be copied one-to-one as a rotation invariant error in the optical surface. The waviness errors on an SPDT-tool stay constant and will repeat exactly in successive machining runs and can be corrected for under the precondition that the machining setup is not changed in the meantime. A-priori measurement of the tool waviness is impractical due to the small size of the tool. As the tool waviness will change as a result of tool wear, correction is limited to this wear rate. Especially in large optics this will play an important role in selection of the machining parameters.

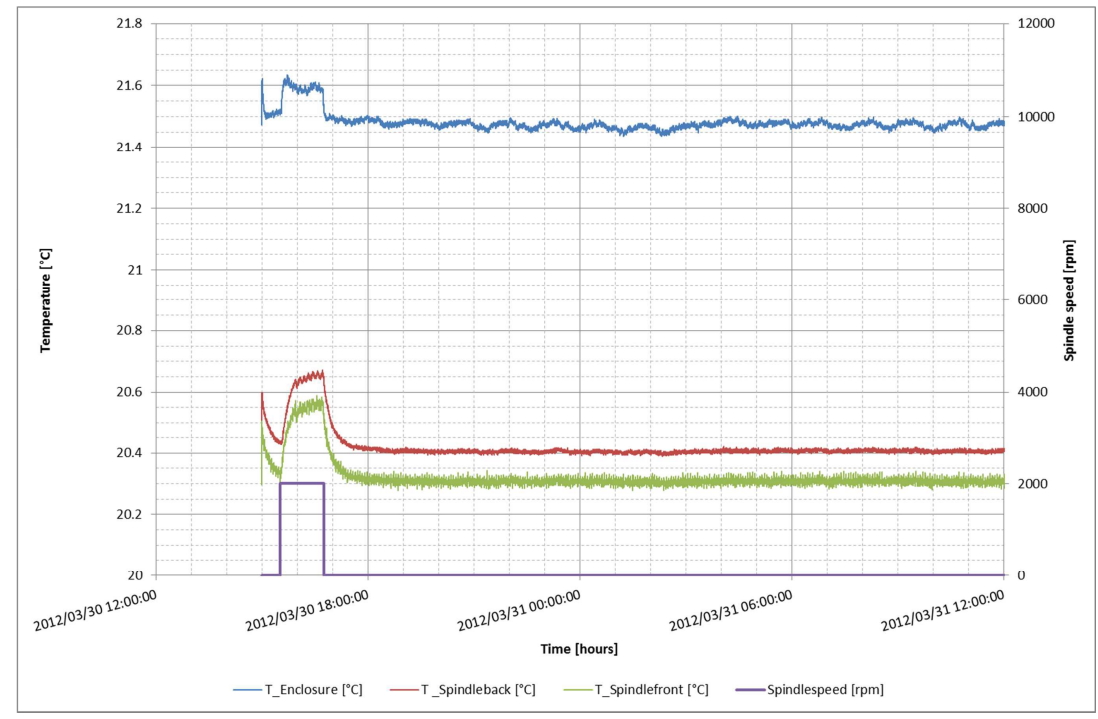

Figure 5: Typical 24 hours temperature cycle of the FF700 from Friday afternoon to Saturday morning is shown.

As mentioned in the previous paragraph corrective SPDT manufacturing can compensate for a number of errors, however a number of error sources cannot be compensated for. Moreover the various error sources cannot be discriminated in the measurement e.g. the contribution of tool waviness or temperature fluctuation to the total deviation of the surface cannot be discriminated. The main goal in our manufacturing strategy is to minimize influences of noncorrectable error sources and correct the other sources.

To minimize thermal influences the temperature the Freeform 700 is equipped with an temperature controlled enclosure to minimize external temperature influences. Both machines are equipped with chillers on the main spindles. 
Additionally on both machines the temperature is monitored at various locations to decide if the machine is thermally stable before starting the machining process.

In Figure 5 a typical 24 hours temperature cycle of the FF700 is shown. The overall thermal balance shows a steady temperature difference of approximately $1{ }^{\circ} \mathrm{C}$ between the enclosure and the spindle. Spinning-up the spindle from 0 to 2000rpm show a steep temperature increase in the spindle in the first half-hour and a slight increase in next hour. It is discussable if the spindle is already stable after 1.5 hour. The rest of the graph shows a stable situation for the spindle during part of the weekend, showing the system in itself is stable when no external influences are present. Especially for freeform optics, which are cut in slow tool servo mode, single machining runs of 20 hours are not uncommon, underlining the necessity of a thermal stable environment during the machining process.

To minimize the influence of clamping, our interface designs are focusing on robustness, decoupling of clamping forces and the optical surface, thereby facilitating minimal deformation during clamping.

Conclusively we can state that the cutting process in itself is very repetitive although with large optical surfaces tool wear becomes an issue. To take full advantage of the repetitively of the SPDT process the machine tool needs to be in perfect condition repetitive errors can be compensated very well, either by on-machine metrology or using dedicated freeform measurement equipment like NANOMEFOS. Non-repetitive errors are mainly introduced by clamping deformations and thermal effects. At TNO there is therefore a strong focus on clever interface design to minimize clamping induced surface errors and monitoring of temperature-stability of the equipment to minimize thermally induced surface errors.

\section{ITERATIVE PROCESSES RESULTS}

\subsection{Manufacturing of the L2 of the Optical Tube Assembly}

For the ESO Very Large Telescope, TNO has made four Optical Tube Assemblies for the Four Laser Guide Star Facility. Each OTA is a large 20x Galilean beam expander, which expands a $\varnothing 15 \mathrm{~mm}, 25 \mathrm{~W} \mathrm{CW} 589 \mathrm{~nm}$ input laser beam to a steerable $\varnothing 300 \mathrm{~mm}$ output. The L2 lens is a $\varnothing 380$ conical convex lens with a radius of curvature of $637 \mathrm{~mm}$ and conic constant $\mathrm{k}=-0.4776$. This results in a departure from the best-fit-sphere of $320 \mu \mathrm{m}$. The required form accuracy is 2 fringes regular and 2 fringes irregular power, and $26 \mathrm{~nm}$ rms of mid-spatials.
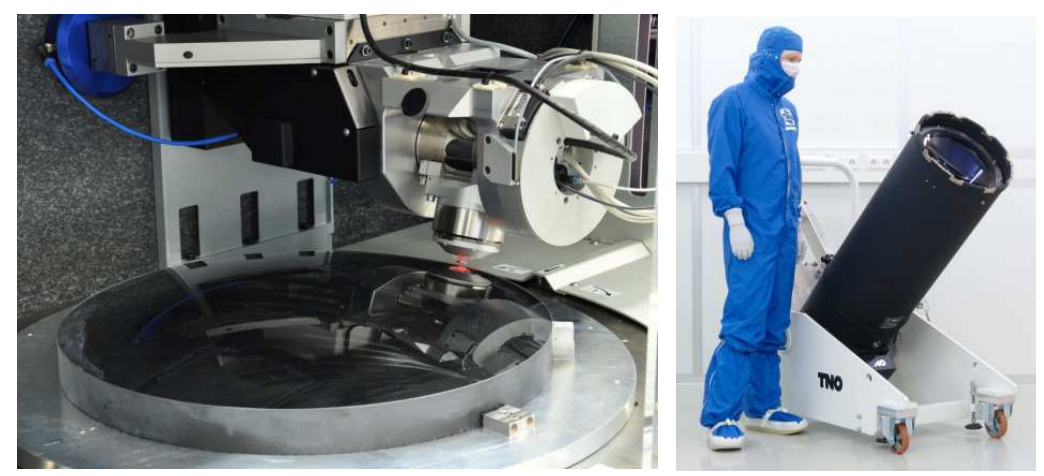

Figure 6: Measurement of the OTA-L2 lens on NANOMEFOS (left) and completed OTA-tube (right)

The OTA L2 aspheres cannot be polished conventionally due to the large departure from the best fit sphere. Deterministic local polishing is therefore applied on the Zeeko CNC polisher. With a diameter of $380 \mathrm{~mm}$, the OTA L2 lens can only be polished in spiral polishing mode on this machine. In the standard precession angle for spiral polishing the rotation of the bonnet is in the same direction as the surface feed, introducing large mid-spatial frequencies in the surface as explained in figure 4. A process optimization was performed to minimize the inherent mid-spatial generation, minimize the roughness and optimize the process predictability.

Figure 7 shows the result of the first L2 lens needed for OTA. The lens blank was ground and pre-polished by our supplier. It can be seen that the initial error is about $5 \mu \mathrm{m}$ PV (incl the radius error), or almost $1200 \mathrm{~nm}$ rms. Next, six iteration runs were needed to reach the required final rms shape error. The determinism in the corrective polishing is relatively good, but because of the tool wear during polishing, the polishing runs needed to be shorter than six hours. The 
final form error is $24 \mathrm{~nm} \mathrm{rms,} \mathrm{of} \mathrm{which} \mathrm{about} 14 \mathrm{~nm} \mathrm{rms}$ is mid-spatial content. A final surface roughness of approximately $1 \mathrm{~nm}$ on the the N-BK7 material was reached.

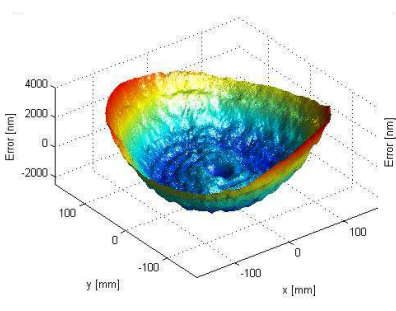

(a) $1198 \mathrm{~nm} \mathrm{rms}$

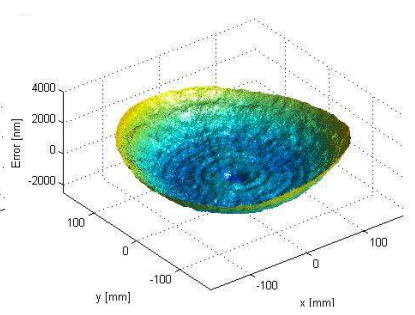

(b) $708 \mathrm{~nm} \mathrm{rms}$

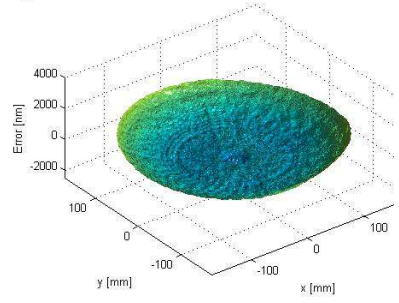

(c) $407 \mathrm{~nm} \mathrm{rms} \quad \Rightarrow$

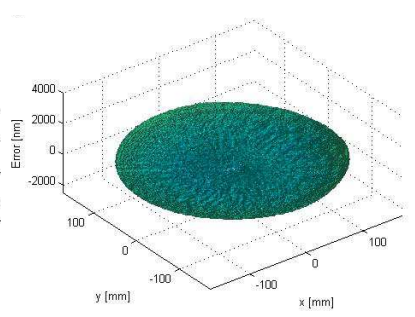

(d) $158 \mathrm{~nm} \mathrm{rms}$

Note: (e)-(g) zoom 10x

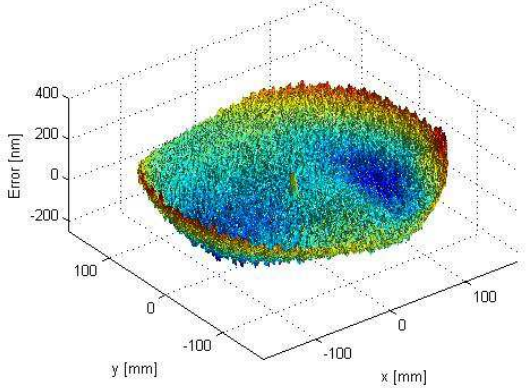

(e) $54 \mathrm{~nm} \mathrm{rms}$

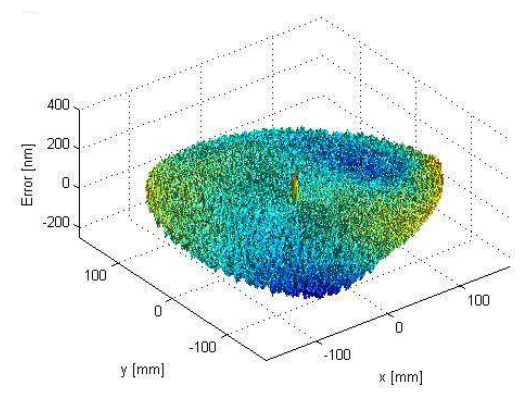

(f) $36 \mathrm{~nm} \mathrm{rms}$

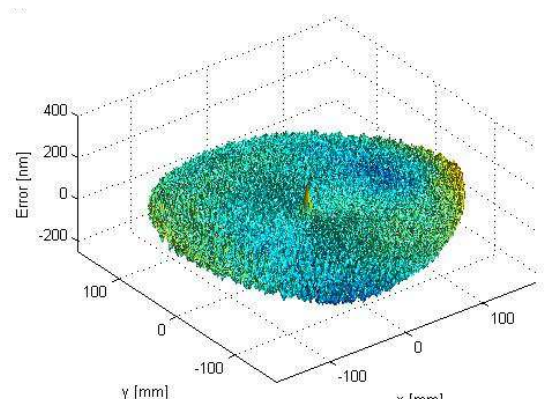

(g) $24 \mathrm{~nm} \mathrm{rms}$

Figure 7: Deterministic polishing result of the OTA L2 lens: (a) shows the pre-polished state as delivered by our blank supplier. Next, in 6 iterations, the total rms error is decreased to $24 \mathrm{~nm}$ over the effective aperture of $330 \mathrm{~mm}$ diameter. The PV error of the lens is $307 \mathrm{~nm}$. In the centre a peak defect can be seen that is the result of cloth wear during the long polishing run. Note that the vertical scale is zoomed 10x for figures (e) to $(\mathrm{g})$

A more detailed description of the manufacturing of the OTA-L2 lens can be found in [4].

\subsection{Corrective SPDT using on-machine metrology}

In October 2011 the first observations with the SCUBA-2 instrument at the 15-metre James Clerk Maxwell Telescope (JCMT) located atop the 4,300-metre high peak of Mauna Kea in Hawaii where made. SCUBA-2 is a sub-millimeter wavelength instrument which will map large areas of sky up to 1000 times faster compared to the original SCUBA camera, with a much larger field-of-view and sky-background limited sensitivity.

In 2003 TNO started with the design and manufacturing of the nine freeform Aluminum mirrors of the SCUBA-2 instrument. The five largest mirrors where iteratively handpolished and measured by a CMM. The four smaller mirrors where single point diamond turned in slow tool servo mode to generate the freeforms. The mirrors were mounted on a special interface plate that allowed for accurate repositioning during the iterative fabrication and off-machine metrology steps. The interface plate was mounted directly onto the spindle and the mating surfaces were themselves diamond turned to remove tilt errors. The whole interface was designed in such a way as to avoid any stress transference to the mirror. A more detailed description can be found in [3].

As mentioned previously, all mirrors went through a high accuracy milling operation before they were either hand polished or diamond turned. The initial mirrors surface, before being diamond turned, had a nominal accuracy of around $20 \mu \mathrm{m}$, but with a scallop height of around $50 \mu \mathrm{m}$. A roughing cut, with an average feed rate of $1 \mathrm{~mm} / \mathrm{min}$ and an average depth of cut of $20-30 \mu \mathrm{m}$, was required to improve the surface finish of the mirror to a level where finer cuts could be made. A typical final finishing cut, with an average feed rate of $0.5 \mathrm{~mm} / \mathrm{min}$ and an average depth of cut of $5 \mu \mathrm{m}$, led to form accuracies between 4 to $6 \mu \mathrm{m}$ PV. Such a fine cut on a $650 \mathrm{~mm}$ diameter surface typically took 18 hours to complete. The surface roughness obtained with this process was between 10 and 15nm RMS, which is much better than that required for sub-millimeter wavelengths. 


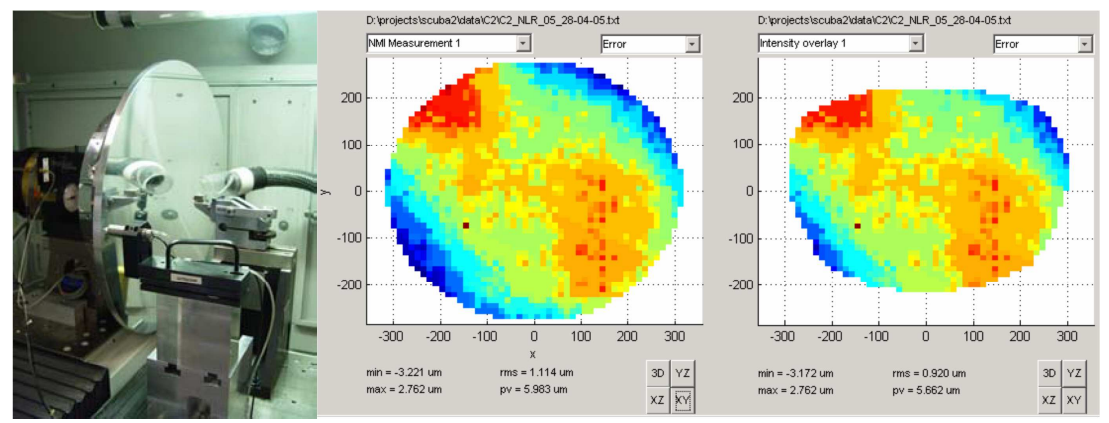

Figure 8: SPDT-Setup for the turning of the SCUBA-2 C2 mirror with a diameter of $650 \mathrm{~mm}$ (left). Error map of the full surface (middle). Error map with reduced aperture (right)

A large emphasis is placed on integrated fabrication and metrology equipment. This type of equipment allows onmachine or in-process metrology, improving efficiency and quality of the process tremendously. TNO, together with help of Precitech, developed such an on-machine metrology system for measuring freeform surfaces directly on the Nanoform 350. The basic system consists of an air bearing LVDT that scans a programmed raster of grid points over the surface. Theoretically, the accuracy attained by such a system should rival the best available CMMs. Figure 8 shows that $5.7 \mu \mathrm{m}$ form PV was obtained on $\mathrm{C} 2$, a mirror of $650 \mathrm{~mm}$ diameter. The LVDT setup is shown in the right of the figure. Nowadays this system is seamlessly integrated in the machine-controller and in daily use for on-machine measurements.

\section{DISCUSSION AND OUTLOOK}

Iterative manufacturing is a very useful method to realize ultra-precision optics for scientific instruments. Two types of iterative manufacturing are available at TNO. CNC controlled sub-aperture polishing and Single Point Diamond Turning. The strategies to use iterative manufacturing for both types of processes differ greatly.

In sub-aperture polishing the form errors are gradually reduced, simultaneously taking into account requirements like surface roughness. The process conditions might be changed in between iteration loops to reach the final requirements. TNO is focusing on process schemes which will efficiently reach the final surface requirements in a robust, reliable and predictable number of iterations.

In SPDT effectively only one iteration cycle or correction cycle is executed. All process conditions, like setup, tooling and cutting parameters are kept constant during this cycle. Only the command positions, used to describe the relative motion between tool and workpiece are modified slightly, based on the measured form deviation. This iteration loop allows for surface precisions in the order of the repeatability of the machine-tool instead of the absolute accuracy of the machine-tool. As a consequence of the given iterative approach in SPDT, the selected machining setup should be such that non-repeatable errors like thermal drift in the machine-tool and deformations due to workpiece clamping etc. are minimized as far as possible.

Currently TNO is working on clamping principles and manufacturing procedures, which can be easily tailored to the job at hand, to reach the next level in diamond turned precision optics.

\section{REFERENCES}

[1] Anderson D., Burge J. "Optical fabrication”, Ch 28, Handbook of optical engineering, Thompson B.J., Malacara D., CRC Press 2001, ISBN: 978-0-8247-9960-1

[2] Henselmans, R., 'Non-contact Measurement Machine for Freeform Optics', PhD Thesis, Technische Universiteit Eindhoven, ISBN 978-90-386-1607-0, 2009

[3] Saunders, I.J., Ploeg, L., Dorrepaal, M.,Venrooy, B. van, "Fabrication and Metrology of Freeform Aluminum Mirrors for the SCUBA-2 Instrument", Proc. SPIE 5869, pp. 14-25 (2005).

[4] Gubbels G., Henselmans R., Drunen C. van, "Flexible manufacturing of large aspheres for VLT's Optical Tube Assemblies”, Proc. SPIE 8126, pp. 81261D-81261D-9 (2011) 